\title{
Should Re-excision Lumpectomy Rates Be a Quality Measure in Breast-Conserving Surgery?
}

\author{
Theresa Schwartz ${ }^{1}$, Amy C. Degnim² ${ }^{2}$ and Jeffrey Landercasper ${ }^{3}$ \\ ${ }^{1}$ Department of Surgery, Saint Louis University School of Medicine, Saint Louis, MO; ${ }^{2}$ Department of Surgery, Mayo \\ Clinic, Rochester, MN; ${ }^{3}$ Norma J Vinger Center for Breast Care, Department of Surgery, Gundersen Health System, \\ LaCrosse, WI
}

In response to recognition of variation in the quality of national healthcare, physicians and their professional societies have emphasized the need for quality measurement and improvement. ${ }^{1}$ Multiple organizations have now developed breast-specific quality measures (QM) to aid quality improvement $(\mathrm{QI}) .^{2-6}$ Many of these $\mathrm{QM}$ have been incorporated into national quality initiatives that provide peer performance comparison and some level of transparency. In addition, during the last decade, the American Society of Breast Surgeons (ASBS), the National Accreditation Program for Breast Centers (NAPBC), and the National Consortium of Breast Centers (NCBC) have defined and endorsed multiple breast-specific quality measures. ${ }^{2-4}$

A QM is an attempt to quantify quality in a domain of care. The most common historical domains of care are "structure of care," "process of care," and outcomes. Recently, "patient centeredness (experience)" and "affordability" have been emphasized as other important domains of quality care. ${ }^{7-9}$ In order to protect against indiscriminate use of metrics that may not be good measures, the federal government has defined $\mathrm{QM}$ as "measures used to assess the performance of individual clinicians, clinical delivery teams, delivery organizations, or health insurance plans in the provision of care to their patients or enrollees, which are supported by evidence demonstrating that they indicate better or worse care." 10 The last phrase is a key part of the definition, indicating that in order for a proposed measure to meet this definition,

(C) Society of Surgical Oncology 2013

First Received: 22 June 2013;

Published Online: 22 August 2013

J. Landercasper

e-mail: JLanderc@gundersenhealth.org there must be supporting evidence that the measure can gauge differences in care.

There are many aspects to the quality of breast-conserving surgery (BCS). These include typical operative outcomes, such as surgical site infection, myocardial infarction, and thromboembolic events. Fortunately, these complications occur so infrequently in BCS that they have limited potential for improvement. ${ }^{11}$ Identifying those areas of BCS that could benefit from quality measurement has recently drawn national attention. Re-excision lumpectomy rates (RELR) after BCS for breast cancer has been discussed as a potential quality measure by the ASBS. ${ }^{1,12}$ However, its use as a quality measure is controversial and is greatly debated. ${ }^{13}$

There are divergent opinions amongst breast surgeons regarding whether to call RELR a QM or whether to use RELR for QI. The primary argument for its use as a QM is strong evidence of significant variability of RELR, ranging from 0 to $70 \%{ }^{14-16}$ On the other hand, the use of RELR as a QM may reduce secondary operations at the cost of unintended harmful changes to other measures of BCS quality. ${ }^{1,12}$ It has also been argued that there is no direct link between RELR and "quality." "This argument is valid if the only quality outcome measured is ipsilateral breast tumor recurrence (IBTR). IBTR has never been demonstrated to differ between patients who require single versus multiple operations to achieve a negative margin. ${ }^{17}$ In a broader view, there may be linkage between RELR and quality. Patient experience and affordability are domains of quality endorsed by the National Quality Forum and others. ${ }^{8,9}$ Both would be expected to improve by lowering the RELR.

Central to the issue of re-excision after lumpectomy is the topic of surgical margins. Surgeons perform re-excision lumpectomies in order to obtain adequate margins, as margin status is associated with IBTR. However, what 
constitutes an adequate negative margin remains unclear, with differing results among many published studies. A recent meta-analysis of 21 BCS studies addressed the relationship between margin status and IBTR after lumpectomy for invasive cancer. ${ }^{18}$ Close to 15,000 subjects were included, mostly with stage I and II breast cancer, with a minimum follow-up of 4 years. In all studies, positive margins were defined as tumor at the inked/ resected edge. Negative margins were defined as no tumor within a defined distance of the resected edge, although the defined distance of negative varied across studies from 1 to $5 \mathrm{~mm}$. The unequivocal finding of the meta-analysis was that a positive margin is not adequate and is associated with increased risk of local recurrence. Among studies evaluating positive/close versus negative margins, odds of local recurrence (LR) were 2.0 for positive/close margins. Among studies that separated the positive and close margin groups, odds of LR were 2.4 for positive margins and 1.8 for close margins compared to women with negative margins ( $p<0.001$ in both instances). Odds of LR remained significantly higher even after adjusting for age, era of study, and use of endocrine therapy.

Regarding the question of adequate size of a negative margin, the data are less clear. The portion of the analysis described above suggests that, similar to positive margins, close margins are also inferior to negative margins. Close margins were defined by the majority of included studies as $<2 \mathrm{~mm}$ or as $<5 \mathrm{~mm}$ (but not positive). Therefore, many women defined as having close margins might have had a negative margin $>1 \mathrm{~mm}$ and possibly even greater than $2 \mathrm{~mm}$. When the authors restricted the analysis to the subset of patients who had negative margins of at least $1 \mathrm{~mm}$, they found that larger negative margins were associated with lower odds of local recurrence-odds ratio (OR) of 0.85 for a $2 \mathrm{~mm}$ margin and 0.58 for a $5 \mathrm{~mm}$ margin, compared to the reference of $1 \mathrm{~mm}$ margin (OR 1.0). However, this trend was not statistically significant $(p=0.11)$ and was further lessened with adjustment for endocrine therapy and a radiation boost. Therefore, in patients receiving appropriate adjuvant therapy, statistically significant differences in LR were not confirmed in the comparison of $1 \mathrm{~mm}$ versus wider negative margins. Limitations of this and other meta-analyses of margin adequacy include the lack of studies specifically comparing ink negative, $1 \mathrm{~mm}$ and $2 \mathrm{~mm}$ margins, reducing the statistical power for detecting differences among various sizes of negative margins. Taken together with the finding that patients with close margins had higher odds of local recurrence, the data in this meta-analysis leaves a lingering question about the effect of "close" margins, specifically those less than $2 \mathrm{~mm}$, on local recurrence risk. In summary, this meta-analysis is helpful in clarifying both the need to re-excise positive margins for invasive cancer as well as the safety of not re-excising margins that are at least $2 \mathrm{~mm}$, as long as appropriate adjuvant therapy is provided that may include a radiation boost. However, the meta-analysis still leaves some question regarding the management of margins less than $2 \mathrm{~mm}$. In a different meta-analysis focusing on DCIS, the odds ratio for LR after lumpectomy for DCIS did not differ for $2 \mathrm{~mm}$ compared to wider margins in patients receiving adjuvant treatment. ${ }^{19}$ Based on these studies, there may be very little difference in what constitutes an acceptable margin width between invasive cancer and DCIS, especially in patients receiving appropriate adjuvant therapies, but the literature is not definitive. The National Comprehensive Cancer Network (NCCN) Guidelines for lumpectomy margins and the margin reexcision algorithm endorsed by the ASBS both reflect the results of the meta-analyses discussed above. ${ }^{12,20}$ The ASBS algorithm is displayed in Figure $1 .^{12}$

How do these data translate into the reality of clinical practice? Not surprisingly, survey studies of breast cancer care providers demonstrate varying opinions on what constitutes an adequate margin for lumpectomy. ${ }^{21-23}$ Two survey studies of surgeons indicated that the majority of surgeons favor a minimum negative margin of at least $1 \mathrm{~mm}$, with $<20 \%$ accepting no tumor at the inked margin. ${ }^{21,23}$ These same surveys identify many care providers who recommend routine re-excision in patients with ink negative or narrow negative margins, a practice not supported by margin meta-analysis or NCCN guidelines. Given these findings, there is potential opportunity to decrease national RELR by simply decreasing the number of patients with initial negative and close negative margin status who routinely undergo re-excision. ${ }^{24}$ Given the available evidence, re-excision of margins greater than or equal to $2 \mathrm{~mm}$ should not be performed routinely. There are several reasons why selective re-excision of a 1-2 mm or greater margin may be indicated. ${ }^{12}$ These include difficulty in pathologic assessment due to a fragmented specimen, residual disease on imaging (such as adjacent malignant appearing calcifications identified on postlumpectomy mammogram), and large volume cancer involvement within 1-2 $\mathrm{mm}$ of the inked margin. In an effort to lower RELR, two surveillance measures regarding margins could be developed to aid identification of practice patterns that contribute to re-excision. These are the percentage of lumpectomy patients with a greater than $2 \mathrm{~mm}$ initial margin free status who undergo re-excision to achieve a wider margin and the percentage of ink positive patients that do not undergo re-excision.

Some variability of reported RELR can be explained by risk adjustment for the factors known to correlate with positive margin status at initial lumpectomy. These factors include method of cancer diagnosis, patient age, tumor size, clinical stage, lobular histology, presence of DCIS, 
and method of histologic processing. ${ }^{12}$ In addition, the completeness of pathology reports also can impact decisions for re-excision. A recent study indicated that 2 years after publication of reporting guidelines from the College of American Pathologists on lumpectomy specimens, approximately half of all reports on breast cancer specimens were not compliant with recommended margin reporting. ${ }^{25}$ Another cause of variability is explained by differing opinions regarding adequate margin width, as discussed above. In two separate surveys, up to $52 \%$ of radiations oncologists and more than $40 \%$ of surgeons endorsed margins greater than $2 \mathrm{~mm}$ and $5 \mathrm{~mm}$, respectively, prior to radiotherapy. ${ }^{21,22}$ Finally, in a recent meta-analysis, the decision to utilize immediate intraoperative margin assessment also correlated with variability of RELR. ${ }^{26}$ Esbona et al. concluded that immediate intra-operative margin assessment with frozen section technique or touch prep cytology compared to no intra-operative pathological evaluation was associated with a lower RELR. ${ }^{26}$

The primary motivation for re-excision after lumpectomy is to reduce cancer recurrence. Hence, re-excision of an ink positive margin represents good quality care because negative margins are associated with lower IBTR. If negative margins could be achieved more often with single compared to multiple operations, then patient centered and affordability domains of quality care could be achieved as well. It is, therefore, relevant to ask whether the use of RELR as a QM helps to achieve this goal. It is also relevant to ask whether unintended harmful consequences may occur if care providers are focused on ensuring their RELR is low, instead of focusing on performing an adequate oncologic operation. If the importance of RELR is elevated too high, would this lead to cases of positive/close margins not being excised, resulting in increased IBTR and ultimately a potential increase in breast cancer mortality? 27 Would women with larger tumors, DCIS, or smaller breasts not be offered BCS due to an increased concern that these patients would likely require a re-excision? Fear of elevated RELR could cause "risk aversion", resulting in increased mastectomy rates and decreased access to BCS. Finally, would too much emphasis on RELR lead to larger resection volumes at first lumpectomy resulting in suboptimal cosmetic outcomes? The relationship of resected breast volume to aesthetic outcome is complex. There is a need for comparative effectiveness research to determine if there are critical differences in patient cosmetic outcome when comparing surgeons who have lower versus higher RELR and when comparing simple to oncoplastic lumpectomy, stratified by breast size, shape and other factors. Comprehensive, validated cosmetic scoring systems, along with patient reported outcomes, such as Breast- $\mathrm{Q}^{\mathrm{TM}}$, need to be incorporated into these studies. ${ }^{28,29}$ The argument against using RELR as a QM is that the unintended consequences described above are possible. If they did occur, they would be detrimental to quality care.

In summary, extreme variability of RELR exists, indicating a performance gap. Reducing variability of national RELR is beneficial because it reduces the financial and nonfinancial burdens of care endured by the patient. However, it is difficult to expect uniform rates of re-excision when a lack of clarity and consensus remains on the definition of an adequate negative margin. Despite the lack of margin clarity, there is opportunity to reduce re-excision rates by ceasing routine re-excision in patients with $2 \mathrm{~mm}$ or greater margins. Since implementing RELR as a QM has the potential to negatively affect other dimensions of breast cancer outcomes, we believe the stewards of measurement of RELR should concurrently audit other aspects of BCS quality to protect against the unintended consequences of mandating reduced re-excision rates. Performance improvement plans to lower re-excision rates will be optimized by multidisciplinary input, collaboration between professional societies, vigilance and an awareness of all possible outcomes.

\section{REFERENCES}

1. Kaufman CS, Landercasper J. Can we measure the quality of breast surgical care? Ann Surg Oncol. 2011;18(11):3053-60.

2. American Society of Breast Surgeons Mastery Program. Available: https://www.breastsurgeons.org/mastery/index.php.

3. National Accreditation Program for Breast Centers Standards Manual. Available: http://napbc-breast.org/standards/standards. html.

4. National Consortium of Breast Centers Quality Measurement Program. Available: http://www.nqmbc.org/about/About.htm.

5. American Society of Clinical Oncology Quality Oncology Performance Initiative. Available from: http://www.asco.org/qopi.

6. Commission on Cancer endorsed Quality Measures. Available: http://www.facs.org/cancer/qualitymeasures.html.

7. Berwick DM, Nolan TW, Whittington J. The triple aim: care, health and cost. Health Aff. 2008;27(3):759-69. doi:10.1377/ hlthaff.27.3.759.

8. National Quality Forum Measure Evaluation Criteria. Available: http://www.qualityforum.org/Measuring_Performance/ Submitting_Standards/Measure_Evaluation_Criteria.aspx.

9. Crossing the quality chiasm. A new health system for the twenty first century. Health care quality may be judged according to its safety, effectiveness, patient-centeredness, timeliness, efficiency and equity. CA Cancer J Clin. 2008;58:266-92.

10. Quality Agency for Healthcare Research and Quality. Tutorials on Quality Measures. U.S. Department of Health and Human Services, 2013. Available: http://www.qualitymeasures.ahrq.gov/ tutorial/varieties.aspx. Accessed 22 May 2013.

11. Neumayer L, Schifftner TL, Henderson WG, Khuri SF, El-Tamer M. Breast cancer surgery in Veterans Affairs and selected university medical centers: results of the patient safety in surgery study. J Am Coll Surg. 2007;204(6):1235-41.

12. American Society of Breast Surgeons. Position Statement on Breast Cancer Lumpectomy Margins, 2013. Available: https:// www.breastsurgeons.org/statements/PDF_Statements/ Lumpectomy_Margins. Accessed 22 May 2013.

13. Morrow M, Katz SJ. The challenge of developing quality measures for breast cancer surgery. JAMA. 2012;307(5):509-10. 
14. McCahill LE, Single RM, Aiello Bowles EJ, Feigelson HS, James TA, Barney T, Engel JM, Onitilo AA. Variability in reexcision following breast conservation surgery. JAMA. 2012;307(5):467-75.

15. Canadian Institute for Health Information on quality of care. Available: http://www.cihi.ca/CIHI-extportal/internet/en/Document/ health+system+performance/quality+of+care+and+outcomes/ outcomes/RELEASE_11OCT12. Accessed 20 June 2013.

16. Jeevan R, Cromwell DA, Trivella M, Lawrence G, Kearins O, Pereira J, Sheppard C, Caddy CM, van der Meulen JH. Reoperation rates after breast conserving surgery for breast cancer among women in England: retrospective study of hospital episode statistics. BMJ. 2012;345:e4505.

17. Coopey S, Smith BL, Hanson S, Buckley J, Hughes KS, Gadd M, Specht MC. The safety of multiple re-excisions after lumpectomy for breast cancer. Ann Surg Oncol. 2011;18(13):3797-801.

18. Houssami N, Macaskill P, Marinovich ML, Dixon JM, Irwig L, Brennan ME, Solin LJ. Meta-analysis of the impact of surgical margins on local recurrence in women with early-stage invasive breast cancer treated with breast-conserving therapy. Eur $J$ Cancer. 2010;46:3219.

19. Dunne C, Burke JP, Morrow M, Kell MR. Effect of margin status on local recurrence after breast conservation and radiation therapy for ductal carcinoma in situ. J Clin Oncol. 2009;27(10):1615-20.

20. NCCN Guideline for breast cancer. Available: www.nccn.org. 2013.

21. Azu M, Abrahamse P, Katz SJ, Jagsi R, Morrow M. What is an adequate margin for breast-conserving surgery? Surgeon attitudes and correlates. Ann Surg Oncol. 2010;17(2):558-63.
22. Taghian A, Mohiuddin M, Jagsi R, Goldberg S, Ceilley E, Powell S. Current perceptions regarding surgical margin status after breast-conserving therapy: results of a survey. Ann Surg. 2005;241(4):629-39.

23. Blair SL, Thompson K, Rococco J, Malcarne V, Beitsch PD, Ollila DW. Attaining negative margins in breast-conservation operations: is there a consensus among breast surgeons? $J \mathrm{Am}$ Coll Surg. 2009;209(5):608-13.

24. Morrow M, Harris JR, Schnitt SJ. Surgical margins in lumpectomy for breast cancer-bigger is not better. $N$ Engl J Med. 2012;367(1):79-82.

25. Persing S, James TA, Mace J, Goodwin A, Geller B. Variability in the quality of pathology reporting of margin status following breast cancer surgery. Ann Surg Oncol. 2011;18:3061-5.

26. Esbona K, Li Z, Wilke LG. Intraoperative imprint cytology and frozen section pathology for margin assessment in breast conservation surgery: a systematic review. Ann Surg Oncol. 2012;19(10):3236-45.

27. Clarke M, Collins R, Darby S, et al. Effects of radiotherapy and differences in the extent of surgery for early breast cancer on local recurrence and 15-year survival: an overview of the randomized trials. Lancet. 2005;366:2087-106.

28. Cardoso MJ, Cardoso JS, Vrieling C, Macmillan D, Rainsbury D, Heil J, Hau E, Keshtgar M. Recommendations for the aesthetic evaluation of breast cancer conservative treatment. Breast Cancer Res Treat. 2012;135:629-37.

29. Cano SJ, Klassen AF, Scott AM, Pusic AL. A closer look at the BREAST-Q®. Clin Plast Surg. 2013;40(2):287-96. 